\title{
SPINDLE CELL CARCINOMA OF THE LARYNX - A DISTINCT CLINICOPATHOLOGICAL AND HISTOLOGICAL ENTITY
}

\author{
Muhammad Faisal ${ }^{1}$, Samaha Nawaz ${ }^{2}$, Omer Waqas ${ }^{2}$, Irfan Haider ${ }^{2}$, Arif Jamshed ${ }^{2}$, Raza Hussain ${ }^{1}$ \\ ${ }^{1}$ Department of Surgical Oncology, Shaukat Khanum Memorial Cancer Hospital and Research Centre, Lahore, Pakistan, \\ 2Department of Radiation Oncology, Shaukat Khanum Memorial Cancer Hospital and Research Centre, Lahore, Pakistan
}

Received: 17 June 2017 / Accepted 05 September 2017

\begin{abstract}
Spindle cell carcinoma (SpCC) is a variant of squamous cell carcinoma with biphasic components and more aggressive behaviour. Its rarity and histopathological pattern pose a diagnostic challenge. Early diagnosis and treatment result in a decrease in local and distant metastasis. Case 1 is a 71-year-old female presented with hoarseness of voice and dyspnoea for 2 years without any risk factors. Fibre-optic laryngoscopy (FOL) revealed smooth polyp hanging from anterior two-third of the left vocal cord. Microlaryngoscopic excision revealed SpCC followed by post-operative radiotherapy and is currently alive. Case 2 is a 72-year-old male presented with worsening stridor for 2 years post-excision of laryngeal nodule and history of smoking and hookah use for $>20$ years. He developed dysphonia after a few months with fixed hard level 3 nodes at the right side. FOL showed a polypoid mass extending from the left vocal cord into the supraglottis. Fine-needle aspiration cytology of the neck swelling confirmed the diagnosis of SpCC. Computerised tomography (CT) chest/abdomen showed distant metastasis. Palliative radiotherapy was given, but the patient died after 3 months due to locoregional failure. Case 3 is a 35-year-old male presented with a history of hoarseness for 3 years with no risk factors. FOL showed a 1.2-cm polypoid growth on the right vocal cord. Total laryngectomy was performed and histopathology showed SpCC. Radiotherapy was given and the patient is alive without disease with regular follow-ups. Smoking and alcohol are thought to be the contributing factors causing this disease. Biphasic nature of the tumour requires pathological sampling for diagnostic confirmation. Surgery combined with radiotherapy has a better survival outcome. SpCC is a rare tumour with a tendency for locoregional recurrence. Surgery should remain the mainstay of treatment followed by post-operative radiotherapy for a better control.
\end{abstract}

Key words: Larynx, radiotherapy, spindle cell carcinoma

\section{Introduction}

Spindle cell carcinoma (SpCC) is a more aggressive and malignant form of squamous cell carcinoma, comprising $2-3 \%$ of all laryngeal cancers. ${ }^{[1-3]}$ Histologically, it has a biomorphic pattern composed of both epithelial and mesenchymal components. Epithelial component comprises of squamous cell (in situ or invasive) and mesenchymal with spindle cells. ${ }^{[4]}$

The carcinoma has male preponderance and occurs more commonly in sixth or seventh decades of life with a 5-year survival of $65-95 \% \cdot{ }^{[5]}$ The prognosis of SpCC is excellent depending on the stage of the tumour and regional nodal

Correspondence: Dr. Muhammad Faisal, Department of Surgical Oncology, Shaukat Khanum Memorial Cancer Hospital and Research Centre, Lahore, Pakistan.

Email:maxfas@live.com metastasis which is seen in one-quarter of the cases and distant metastasis occurs in $5-15 \% .{ }^{[6]}$ The most common site of distant metastasis is the lung, followed by soft tissues and other viscera. The rate of local recurrence is 7-26\%. In most instances, the relapses occurred within 12 months of initial treatment. ${ }^{[7]}$

The calamity of the disease is a difficult diagnosis and definitive management to avoid recurrence. In this case series, we have discussed the clinicopathological aspects of four cases of SpCC along with its management.

\section{Case Report}

\section{Case 1}

A 71-year-old Bengali woman presented at head and neck clinic. The presenting complaints were hoarseness 
of voice and occasional difficulty in breathing for the past 2 years. She had no history of smoking or any other addiction. Her flexible fibre-optic laryngoscope (FOL) examination showed a large smooth surface polyp hanging from anterior two-third of the left vocal cord with both cords being symmetrically mobile. Later that month, microlaryngoscopic excision was performed and histopathology revealed SpCC [Figure 1]. The postoperative magnetic resonance imaging (MRI) scan showed nodularity along the left vocal cord without significant neck nodal disease with the American Joint Committee on Cancer (AJCC) stage-I (T1N0M0). She received hypofractionated radiotherapy of 55 Grey in 20 fractions ( $275 \mathrm{cGy} /$ fraction). The patient did well and went back to her country and is alive till date.

\section{Case 2}

A 72-year-old male presented at the head and neck clinic with worsening hoarseness for the past 2 years. He had been smoking cigarettes and hookah for the past 20 years. He started his treatment 6 months earlier with excision of laryngeal nodule performed at another centre. Histology of the surgical specimen was inconclusive at that time. He developed dysphonia again after a few months, for which he was brought to our institute. Clinically, he had a hard and fixed nodal swelling at level III on the right side of the neck. FOL showed a polypoid mass extending from the left vocal cord into the supraglottis. Fine-needle aspiration of the neck swelling was done which confirmed the diagnosis of SpCC. Computerised tomography (CT) neck showed nodular thickening of the left vocal cord extending up to the anterior commissure. There is a evidence of supraglottic extension with the involvement of ipsilateral aryepiglottic fold, resulting in obliteration of pyriform fossa, while CT-chest/abdomen and chest $\mathrm{x}$-ray confirmed the presence of extensive pulmonary, pleural and hepatic metastatic disease [Figure 2]. Meanwhile, the patient developed dyspnoea and stridor, for which emergency tracheostomy was done. Palliative radiotherapy of $20 \mathrm{~Gy}$ in 5 fractions was given and he died after 3 months due to progressive local and distant disease.

\section{Case 3}

A 35-year-old male presented at radiation oncology department with complaints of hoarseness for the past

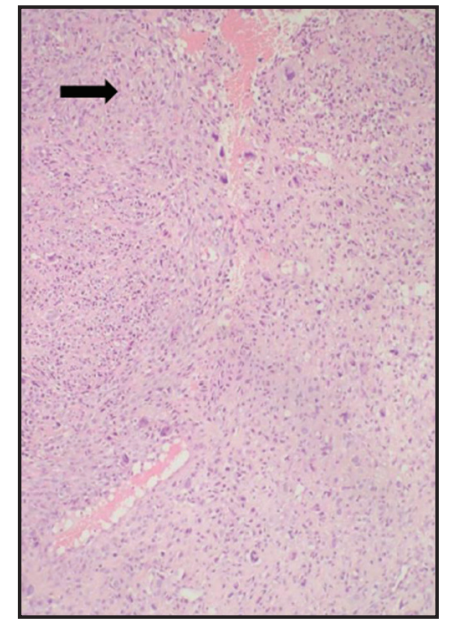

Figure 1: Low power magnification $(\times 10)$ showing tumour comprising interlacing bundles and fascicles (indicated by arrow)

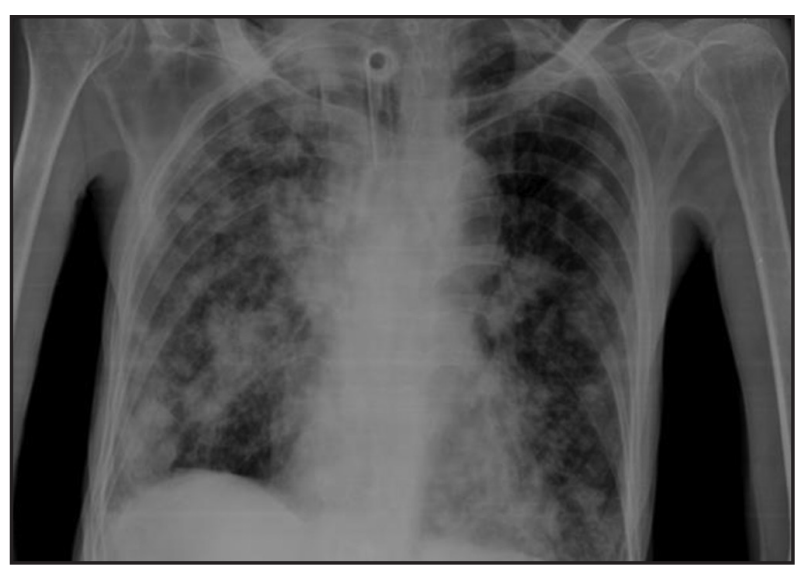

Figure 2: Chest X-ray showing lung metastasis

3 years. There was no history of any addiction reported. FOL showed a $1.2-\mathrm{cm}$ polypoid growth on the right vocal cord. Lymph nodes were not palpable. Excision biopsy done at another centre showed high-grade sarcoma. CT neck also showed T3 disease due to paraglottic extension with bilateral tiny sub centimetre radiologically insignificant lymph nodes. Thus, a total laryngectomy was performed and biopsy revealed SpCC. On gross examination, the larynx contained a polypoid well-circumscribed tumour measuring $1.2 \mathrm{~cm} \times 1.0 \mathrm{~cm} \times 0.6 \mathrm{~cm}$, present on the right side involving the glottic area and extending into the subglottis. Histological examination revealed a tumour showing foci of squamous cell carcinoma in situ with focal invasive component [Figure 3]. Bulk of the tumour was formed by spindle cells with elongated nuclei and 


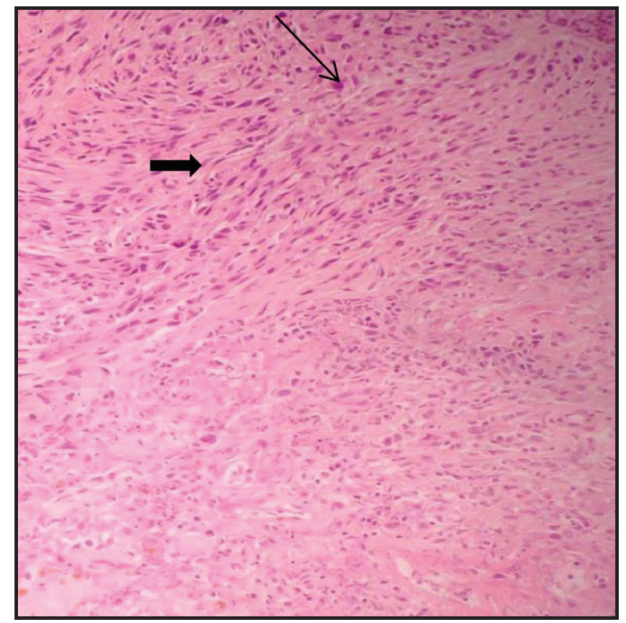

Figure 3: Squamous cell carcinoma with sarcomatoid features comprising spindle-shaped tumour cells (indicated by thick arrow) and markedly pleomorphic cells (thin arrow). $\mathrm{H}$ and $\mathrm{E}$, $\times 20$ magnification

these cells were negative for CK5/6. However, CK5/6 was positive in epithelial component.

These findings favoured carcinosarcoma (SpCC). The tumour invades into the paraglottic space. All resection margins were free of tumour. A radiation dose of 50 Gy in 20 fractions was given to neck including primary and bilateral cervical nodes. The patient has maintained regular follow-up visits and is free of disease till date.

\section{Discussion}

$\mathrm{SpCC}$ is a rare and highly malignant variant of squamous cell carcinoma. It has been referred to by a number of terms such as pseudo-carcinoma, pleomorphic carcinoma, collision tumours, combination tumours, composition tumour, Lane's tumour and polypoid carcinoma. ${ }^{[8]}$ This is explained by the fact that the true nature of the disease has remained controversial for a long time. The incidence is $2-3 \%$ of all laryngeal cancers. The real cause, like most of the other carcinomas, is not known. However, the risk factors include cigarette smoking and alcohol consumption. Many cases reported a history of radiation exposure, but there is no definitive evidence to prove this hypothesis. ${ }^{[9-11]}$

The SpCC more commonly involves glottis than any other subsites in the larynx. Therefore, clinically, patients present with complaints of hoarseness, dysphonia, dysphagia and foreign body sensations in the throat.
Mostly, tumours are pedunculated, exophytic or polypoid in clinical examination causing obstructive respiratory symptoms at an early stage leading to prompt diagnosis and treatment of the tumour. One-quarter of patients presents with nodal metastasis, and distant metastasis is seen in $5-15 \%$. One of our patients presented not only with nodal metastasis but also with extensive lung and liver metastasis which signify the appropriate staging workup before the start of radical treatment.

Pathological examination of the biopsy specimen leads to definitive diagnosis. The tumour has a biphasic pattern composed of both carcinomatous and sarcomatoid component. Typically, a confirmation is achieved by the observation of surface epithelial cells with underlying sarcomatoid spindle-shaped neoplastic proliferation. In case of surface ulceration or denuded epithelium, the dignity remains undefined and diagnosis of $\mathrm{SpCC}$ is difficult to render. The pathogenesis of SpCC is controversial and its derivation has remained ambiguous till date. Although there are no large mass studies available that suggest the epithelial origin of the tumour, a few histological theories are certain in favour of it including (1) its location and occurrence which are at the site of squamous epithelium, (2) neoplastic mesenchymal spindle cells occurring in association with nests of benign or malignant squamous epithelium, (3) there polyploid nature and (4) immunohistochemical analysis shows reactivity to epithelial antigen. However, there are many evidence that demonstrate metastasis of both sarcomatoid and epithelial components in a single lymph node or separate metastasis of individual component to various sites, suggesting that sarcomatoid spindle cells are not just a metaplastic reaction to overlying malignant epithelium rather it's an independent morphology fully capable of having its own natural course.

In addition to histological studies, immunohistochemistry also demonstrates two distinct pathologies. The epithelial component is positive for a panel of keratin markers (AE1/AE3 'CK1'), epithelial membrane antigen, $\mathrm{K} 1$ and $\mathrm{K} 18$, and the mesenchymal component is positive for variable smooth muscle actin, S-100 protein, musclespecific actin, desmin-D33 and desmin-DR11. However, both components are positive for vimentin; however, it is more expressed in sarcomatoid component of the tumour or in those epithelial cells that show metaplastic mesenchymal transformation while losing their ability to react with 
epithelial markers. p53 is an epithelial cell proliferation and differentiation marker and is important in confirming the diagnosis of $\mathrm{SpCC}$ of the head and neck region.

SpCC is staged according to AJCC TNM staging system based on tumour size and presence or absence of locoregional lymph node and distant metastasis.

There is no consensus available yet on treatment modality of SpCC. The goals of the treatment include cancer cure, organ preservation, good voice quality and low risks of complications. Surgery and radiation are the two treatment options available, but surgery remains the mainstay of the treatment. The choice depends on the stage and location of tumour. For small stage tumours T1 and T2 polypectomy, microlaryngoscopic excision or vocal cord stripping followed by adjuvant radiotherapy is considered as the treatment of choice. However, Thompson et al., in their study, showed that patients who were managed by surgery alone had a better outcome than the patients who were treated with surgery and adjuvant radiotherapy. The group of patients who were treated with radiation alone and those who were treated with radiation and surgery both had a better survival (3.6 years) compared to those who were treated with surgery alone (1.9 years). Theoretically, some studies also show that the use of radiation therapy in SpCC is not suggested because mesenchymal component of the disease is highly resistant to radiation. For advance, high stage ( $\mathrm{T} 3$ and $\mathrm{T} 4$ ) and recurrent tumours, a more aggressive approach is adapted including hemilaryngectomy or total laryngectomy with or without lymph node dissection followed by post-operative radiotherapy ${ }^{[12-15]}$ In one of our case reports, advance SpCC with extensive liver and lung metastasis is discussed in which salvage surgery and palliative radiotherapy were adopted which showed the survival of 2 years. Radiation alone might not be the control of the disease, but, when combined with salvage surgery, it has a survival of $79-98 \%$ in recurrent diseases.

\section{Conclusion}

$\mathrm{SpCC}$ is a rare but aggressive variant of squamous cell carcinoma. As there are chances of local as well as distant metastatic disease, so cross-sectional imaging of lungs and liver should be incorporated in routine staging workup. Origin of the tumour remains controversial so is its definitive mode of treatment. With our case series, we conclude that surgery must be the mainstay of treatment, but adjuvant radiation should be combined to have good locoregional controls.

\section{Conflict of Interest}

The authors declare that they have no conflict of interest.

\section{References}

1. Völker HU, Scheich M, Höller S, et al. Differential diagnosis of laryngeal spindle cell carcinoma and inflammatory myofibroblastic tumor report of two cases with similar morphology. Diagn Pathol 2007;2:1.

2. Anderson CE, Al-Nafussi A. Spindle cell lesions of the head and neck: An overview and diagnostic approach. Diagn Histopathol 2009;15:264-72.

3. Thompson LD. Squamous cell carcinoma variants of the head and neck. Curr Diagn Pathol 2003;9:384-96.

4. Zarbo RJ, Crissman JD, Venkat H, et al. Spindle-cell carcinoma of the upper aerodigestive tract mucosa. An immunohistologic and ultrastructural study of 18 biphasic tumors and comparison with seven monophasic spindle-cell tumors. Am J Surg Pathol 1986;10:741-53.

5. Thompson LD, Wieneke JA, Miettinen M, et al. Spindle cell (sarcomatoid) carcinomas of the larynx: A clinicopathologic study of 187 cases. Am J Surg Pathol 2002;26:153-70.

6. Lewis JE, Olsen KD, Sebo TJ. Spindle cell carcinoma of the larynx: Review of 26 cases including DNA content and immunohistochemistry. Hum Pathol 1997;28:664-73.

7. Lambert PR, Ward PH, Berci G. Pseudosarcoma of the larynx: A comprehensive analysis. Arch Otolaryngol 1980;106:700-8.

8. Miyahara H, Tsuruta Y, Yane K, et al. Spindle cell carcinoma of the larynx. Auris Nasus Larynx 2004;31:177-82.

9. National Cancer Institute. Cancer Facts. Head and Neck Cancer: Questions and Answers; 2012. Available from: https//www.cancer.gov/types/head-and-neck/head-and-factsheet. [Last accessed on 2017 May 01].

10. Anand P, Kunnumakkara AB, Sundaram C, et al. Cancer is a preventable disease that requires major lifestyle changes. Pharm Res 2008;25:2097-116.

11. Aggarwal BB, Vijayalekshmi RV, Sung B. Targeting inflammatory pathways for prevention and therapy of cancer: Short-term friend, long-term foe. Clin Cancer Res 2009; 15:425-30.

12. Luna-Ortiz K, Mosqueda-Taylor A. Supracricoid partial laryngectomy as a primary treatment for carcinosarcoma of the larynx. Ear Nose Throat J 2006;85:337-41.

13. Stomeo F, Rocca PC, Bozzo C, et al. Laryngeal true malignant mixed tumor. Head Neck 2009;31:556-60.

14. Ianniello F, Ferri E, Armato E, et al. Carcinosarcoma of the larynx: Immunohistochemical study, clinical considerations, therapeutic strategies. Acta Otorhinolaryngol Ital 2001;21:192-7.

15. Marioni G, Bottin R, Staffieri A, et al. Spindle-cell tumours of the larynx: Diagnostic pitfalls. A case report and review of the literature. Acta Otolaryngol 2003;123:86-90. 